\title{
Thermal properties of highly structured composite and aluminium sheets in an aerodynamic tunnel
}

\author{
Petr Kulhavy ${ }^{1, a}$, Josef Egert ${ }^{1}$ \\ ${ }^{1}$ Faculty of Mechanical Engineering, Technical University of Liberec, Studentská 2, 461 17, Liberec 1, Czech Republic
}

\begin{abstract}
This article deals with the thermodynamic behaviour of heat shields - structured metal and composite plates. Experiments have been carried out in a wind tunnel with an additional heating, which simulates the heat source from engine or exhaust pipe and simultaneously the airflow generated during a car movement. The tested sheets with hexagonal structure were a standard commercial made of aluminium and a second manufactured by replication (lamination, diffusion) from glass fabric. The airflow in a parallel way along the sheets was analysed experimentally in order to determine the heat transfer efficiency between surfaces of sheets and surrounding airflow. The temperature on the sheets was chosen to observe the effects of different sheets material, various heat power and airflow velocity. During the experiment a thermal input below the sheets and airflow velocity through the tunnel have been changed. The thermal field distribution on the metal sheet is different than in case of composite sheet. For the composite material the thermal field distribution was more homogeneous. This article describe briefly also methods of obtaining real composite geometry based on scanned data and their reconstruction for using in some future numerical models.
\end{abstract}

\section{Introduction}

Due to the high energy demand and rising prices of materials plays the reduction of weight an important role through almost all industries. For vehicles it is necessary to minimize weight of parts without affecting their functionality and safety - often even on the contrary with their improvement. According to [1] an vehicle weight reduced by $100 \mathrm{~kg}$ saves 0.25 to $0.51 / 100 \mathrm{~km}$ of fuel that means reducing of $\mathrm{CO}_{2}$ emissions from 3.5 to $8.4 \mathrm{~g} / \mathrm{km}$. Thin structured metal sheets (Fig. 1) become still more important for lot applications. One of their possible using could be as heat shields over the car exhaust and engine [2]. Their function is to create a thermal barrier between parts which should stay cool even if they are affected by high temperature radiation from a near heat sources [3]. The structured sheets offer us for particular applications the possibility of influencing on mechanical parameters, not only in terms of stress distribution, but also change in stiffness, shifting of natural frequencies or even customization of aerodynamic properties. They are used as a semi-finished product $[4,5]$. From the point of the heat transfer mechanism, the structured surfaces intensified the convection heat transfer rate due to higher surface area and the suitable flow behaviour around the structured metal sheets as referred in $[5,6,7]$ their papers focuses on heat shield application which is typically used in automotive.

For more efficient decreasing of the weight have been nowadays used also composite materials, which have considerably better specific strength than conventional. When compared with metal materials, composites are more appropriate especially in the terms of an efficient

\footnotetext{
${ }^{\mathrm{a}}$ Corresponding author: petr.kulhavy@tul.cz
}

material using and saving weight. For this reason, composite parts whose properties could be modified directly with regard to the specific application, that significantly increases the utilization efficiency of materials are often used. Composite parts could be produced in many ways as winding, wrapping, pultruding or continuous laminating. Highly structured composite parts introduced in this work have been created by the method of steel templates replication by lamination and difusion method [8].

This article deals with experimental comparison of thermal transfer between steel and composite - glass fibre sheets, experimentaly measured in a wind tunnel with an additional heating, which simulates the heat source from engine or exhauster and simultaneously the airflow generated during driving a car.

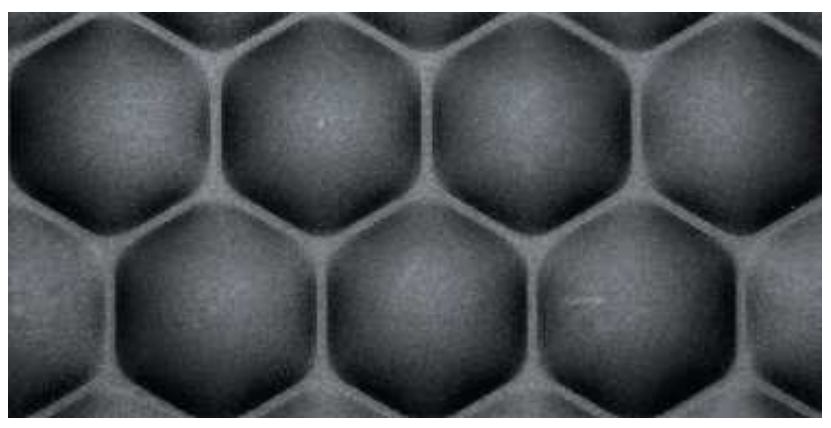

Figure 1. The hexagonal sheet structure 


\section{Used materials}

Using of the structured sheets (Fig. 1) could be as the heat shields over the car exhaust and engine, cover of movable parts, reflective layer of lamps or even a drum of wash machines. The replicated parts are structured glass or carbon composites created by replication of steel templates. Production of steel templates is a technological procedure mostly based on pressing or rolling $[4,5]$.

This way structured metal sheets were used as the shape template for replication on fiber fabrics, because it isn't possible produce such structure composite parts by a direct way. So, in this case instead of finding complicated methods for direct manufacturing composite parts, an sub step has been done with using the technologically simpler steel plate. Long fiber composites offer generally the greatest flexibility for design specification. However, the metal materials, have still lower price, also have great potential due to the possibility of they recycling and excellent technological processability [8].

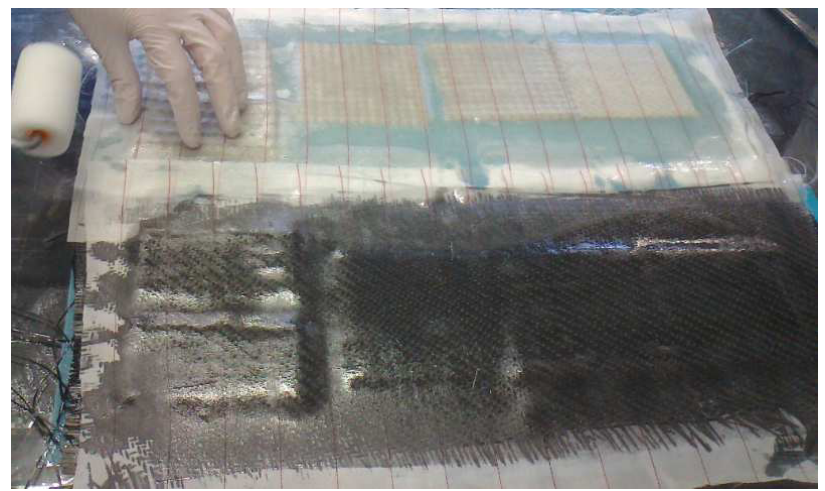

Figure 2. The replication - manual saturation of plies

\subsection{The tested prototype}

An unilateral replication by stamping fabrics over the aluminium structure was carried out to create the FRP samples. Chemically treated surfaces of the aluminuim sheets were covered with four layers of glass or carbon fabrics (Fig. 2) and gradually saturated with an epoxy matrix. The created structure was uniformly without visible defects. Surely, according to $[9,10]$ the material properties determined from standard specimens tested in laboratory may often significantly deviate from components manufactured in factory.
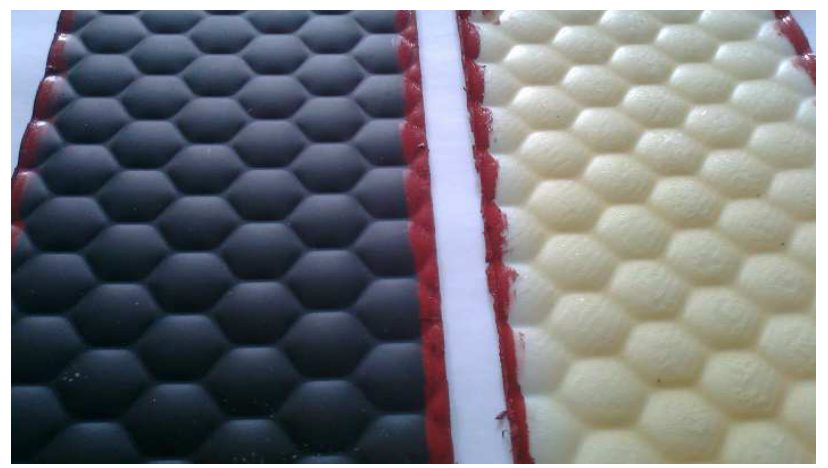

Figure 3. The original and composite part

\subsection{Digitization and meshing of samples}

In contrast to a conventional metal parts, for the composite materials we are usually not able to accurate predict the final shape and dimensions of manufactured parts. This is caused by the fact, compared with e.g. precise machining, in this case we could speak moreover about copying shapes of used molds. Also the interleaving of individual plies including degree of resin saturation is not always the same and temperature or pressure could also differs. It means, we are not able to have the accurate and real CAD models, before we physically create the part. For the composite plate replicated by the above described method we need to know the exact dimensions and shape in order create a numerical model for simulation. For this reason the replicated plates have been scanned. At this moment, we got only a giant cloud of points that present just an undefined surface (Fig. 4 blue).

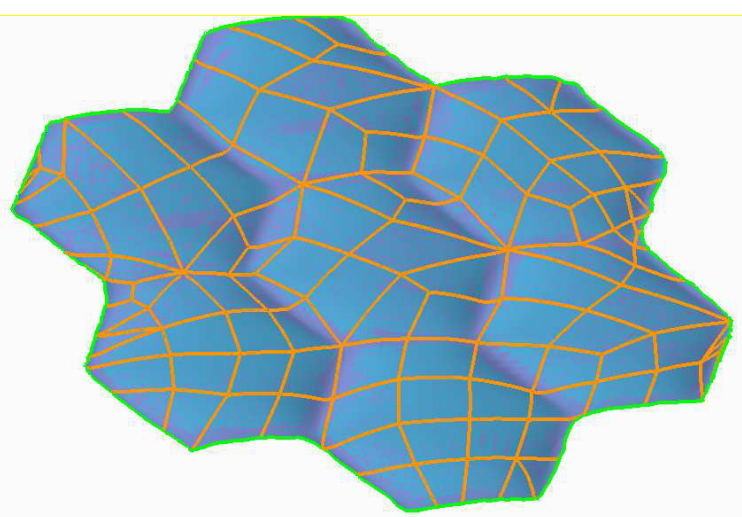

Figure 4. Part of the scanned and consequently styled geometry

To provide possibility import of a such data structure into a classic CAD their manufacturers have usually some own tools. In our case PTC Creo - Import data doctor has been used. These module contain algorithms for an additional editing, smoothing and cleaning of the imported meshes. It is also possible to cover the gaps, closed imperfect shapes or trim the surfaces. After the "coarse" repairing of the mesh we obtain a parametrically defined surface (Fig. 4 orange lines). Now, the CAD system is able to work with this way optimized data and use it as a design element for the projection, intersection, references or definition points, tangent properties etc.

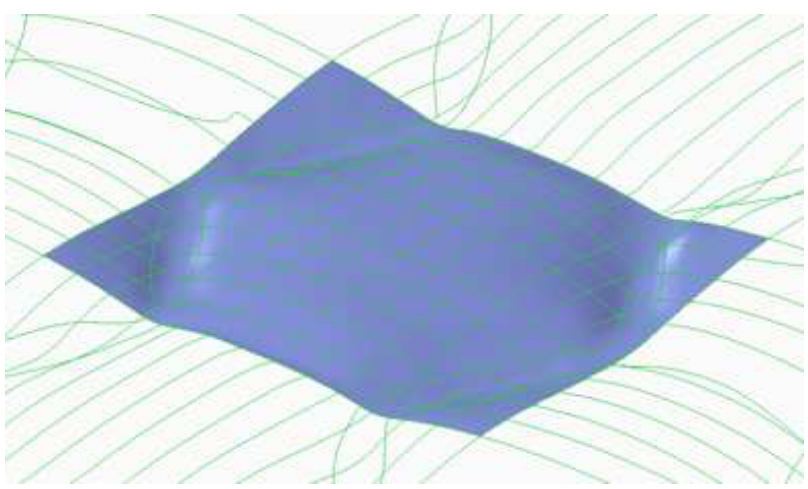

Figure 5. Boundary blend based on intersection surfaces 
The most precisely defined fragments in a NURBS format, which are suitable for CAD data we could get by local cut outs and creation of a boundary blend as is seen in Fig. 5.

At this moment a real problem is that all the inaccuracies that arise during scanning and also geometric deviations of the measured parts are reflected and stacked up. Some unmeasurable errors in the intersection of supporting curves of created entities arise there almost always and this leading to a total or local collapse of the geometry. Sometimes, like in our case the inaccuracies are stacked and deform "only" the real shape locally - e.g. some spurs or holes could arise in the geometry as could be seen in Fig 6. About the reconstruction of similar geometries and their meshing it is possible to read more e.g. in $[11,12]$.

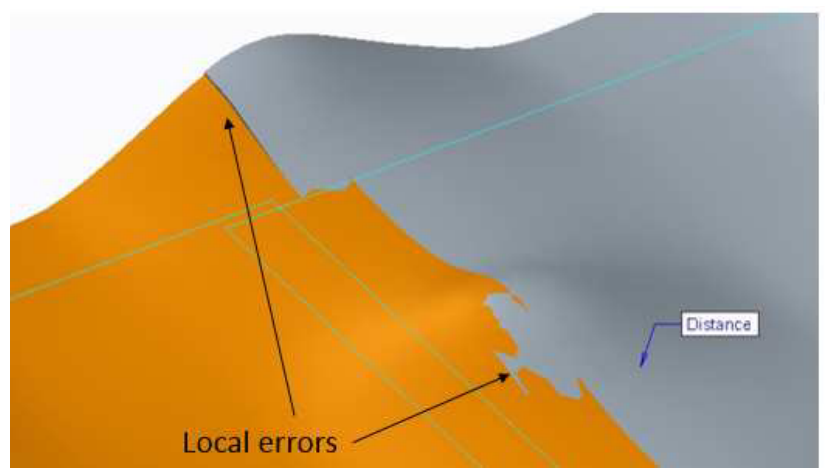

Figure 6. Local errors caused by stacking of inaccuracies

Now, probably the best variant how to repair the erroneous areas is to set them boundaries and fill with vector field consequently interleaved by surface. For the required vector definition was possible to simply use the adjustable parameters of normality, tangentiality or parametric settings of the transitions.

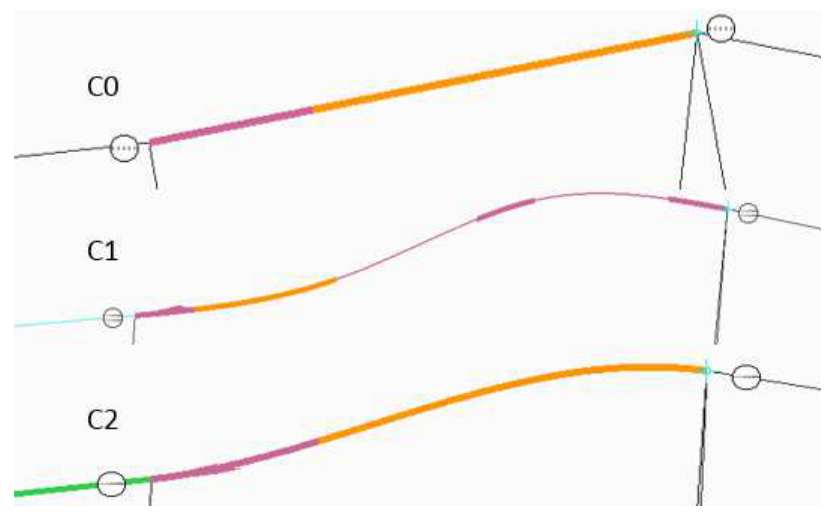

Figure 7. Classes $n$ of the continuity transitions between individual segments

A very important parameter is settings of the parametric classes $n$ of the continuity transitions between individual segments that defines us 3 classes of continuous. Connection $\mathrm{C}^{0}$ means in common point two arbitrarily passing vectors and two curves with different radius of curvature. Continuity $\mathrm{C}^{1}$ is characterized by the one common tangent vector $\overrightarrow{t_{l}}$ in the transition point, but different radii of curvature. Completely analogously then the last class $\mathrm{C}^{2}$ provides a common tangent vector and value of the radius of curvature in the transition point as could be seen in the Fig. 7. Then, based on this surfaces, fully active CAD model could be created and exported to a required format. Six examples have been created (Fig. 8) in order to find out the dependency of geometric reconstruction (continuity vectors degree) and parametres of the computational mesh.

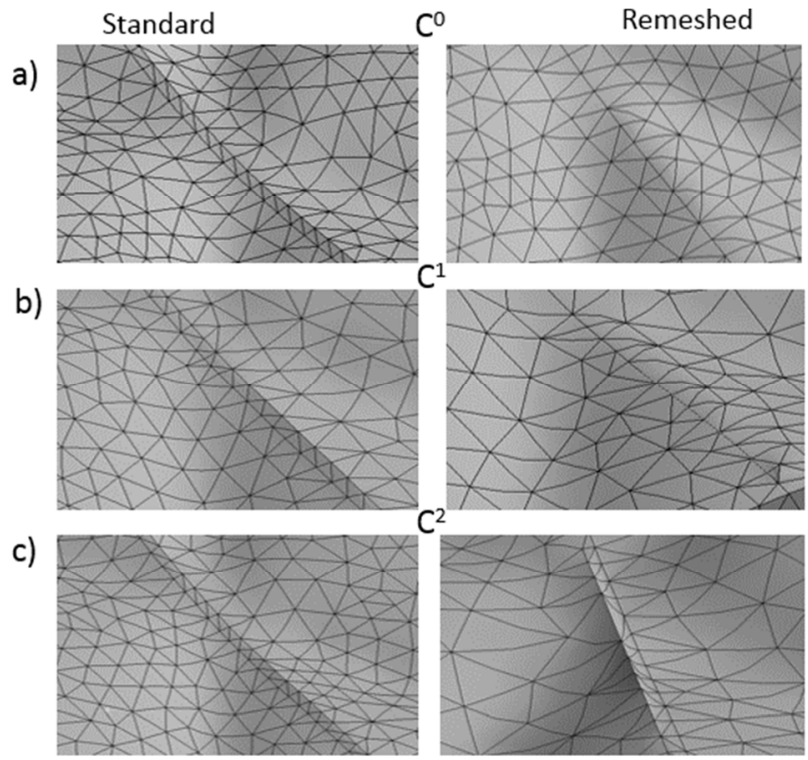

Figure 8. The mesh dependency on the vector continuity

The mesh dependency on the geometric vector continuity was compared in the commercial sw Ansys. First case was an automatically generated a tetrahedral mesh with $+80 \%$ relevance of fine mesh (Fig. 9 Standard). In the second case the mesh was optimized with just several node and curve pinches and refined with virtual cells quite easily and fast. The results could be seen in Fig. 9 (column remeshed) or mesh the statistics in the Tab. 1. As could be recognized from the Table and Figure, the high degree of geometry optimization does not have the desired effect to the mesh quality and even just for the coarse transmission $\mathbf{C}^{\mathbf{0}}$ we could obtain with using some remeshing tools almost equal quality with a considerable smaller effort.

Table 1. Mesh statistics

\begin{tabular}{|c|l|c|c|c|}
\cline { 2 - 5 } \multicolumn{1}{c|}{} & Method: & $\mathrm{C}^{0}$ & $\mathrm{C}^{1}$ & $\mathrm{C}^{2}$ \\
\hline \multirow{2}{*}{$\begin{array}{c}\text { Standard } \\
\text { mesh }\end{array}$} & $\begin{array}{l}\text { Nr. of } \\
\text { elements }\end{array}$ & 9576 & 10268 & 10017 \\
\cline { 2 - 5 } & $\begin{array}{l}\text { Nr. Of } \\
\text { nodes }\end{array}$ & 17261 & 18316 & 17959 \\
\hline \multirow{2}{*}{ Remeshed } & $\begin{array}{l}\text { Nr. of } \\
\text { elements }\end{array}$ & 9427 & 8603 & 9788 \\
\cline { 2 - 5 } & $\begin{array}{l}\text { Nr. of } \\
\text { nodes }\end{array}$ & 17024 & 15647 & 17576 \\
\hline
\end{tabular}




\section{Experiment}

The aim of the carried tests was to assess the thermodynamic behaviour for composite materials. In this research the results obtained with composite prototypes were compared with results obtained in previous research for steel prototype with the same structure shape [3]. The experimental device (Figure 9, $10)$ is combination of an open aerodynamic tunnel with a resistive heater located inside the box. Tested sample is placed on the bottom of test section of aerodynamic tunnel on the top of heater box.

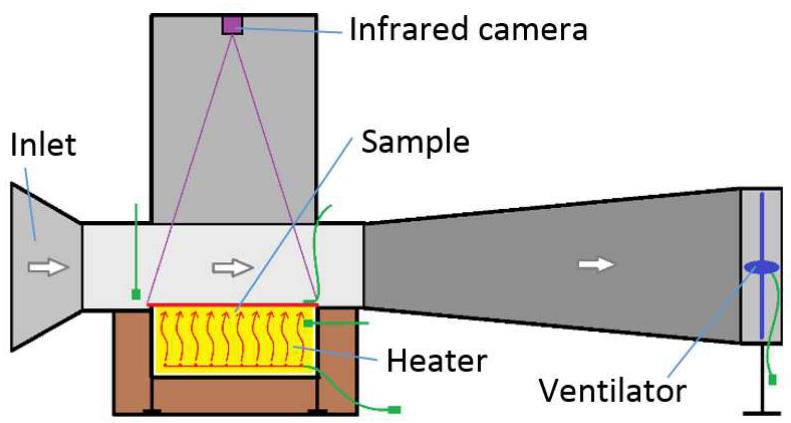

Figure 9. The experimental device

Detailed description of the device is available in previous study [3]. At the inlet of the model, there is a confusor with rectangular cross section which sucks the ambient air into the tunnel. The airflow speed was measured by a hot wire anemometer probe in front of the test section that is located near the bottom side of the tunnel. One side of the sheet was cooled by the airflow and the other side was heated by the convection from the hot air and by the heat radiation from heater. The global temperature field on the sheet surface was measured by the infrared camera placed on the top of the vertical part of the tunnel and locally with thermocouple. To minimize heat losses, the heating box was isolated by a polystyrene, mineral cotton, calcium-silicate layer and aluminium foil. The temperature inside the heat box was regulated varying voltages and measured with the thermocouple. The ventilator rotational speed was controlled by a potentiometer.

The tested sample was measured in the same way as in previous research to obtain comparable results. Sample was measured in the heating power range from $100 \mathrm{~W}$ to $500 \mathrm{~W}$ with step of $100 \mathrm{~W}$ and Reynolds numbers varied from 10000 to 145000 . The Reynolds number range was given by the airflow speed which was limited by the power of used axial ventilator. The range of the heating was limited by range of infrared camera. For each of investigated state an infrared image was taken and the average and maximum temperature of the sheet s were determined. Also, the temperature inside the heating box and ambient temperature were recorded. The thermal camera emissivity for the lacquered aluminium sheet was based on the camera manufacturer tables and verified by measurement with thermocouples set to value 0.9 and for the epoxy composite to 0,85 .

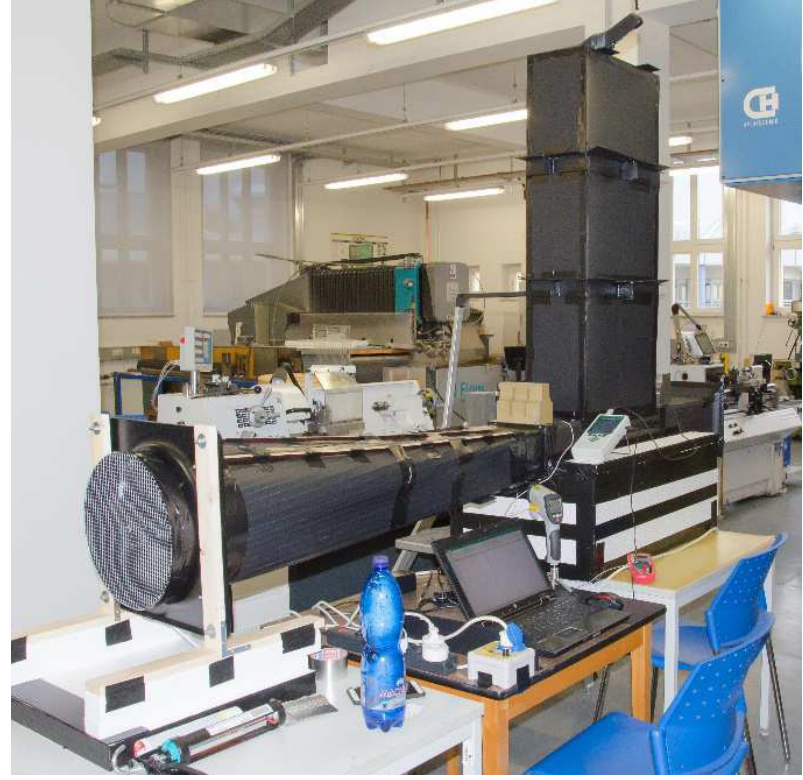

Figure 10. Assembly of the real experimental device

Velocity profile in the inlet of aerodynamics tunnel was measured with anemometric probe. Velocity was set to $13 \mathrm{~m} / \mathrm{s}$ with probe in default probe position used for other regimes and then probe was moved across the cross section. Results are shown in Figure 11. Lover velocity near bottom wall is probably caused by the lead edge before the tested sample. Velocity profile in the rest of cross section is balanced.

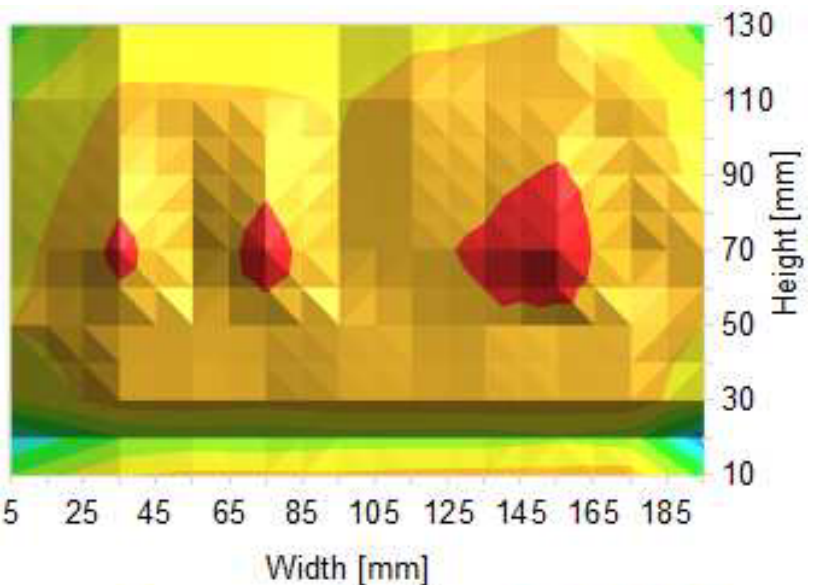

- $10,00-10,50=10,50-11,00=11,00-11,50$

$=11,50-12,00=12,00-12,50 \quad 12,50-13,00$

$=13,00-13,50=13,50-14,00$

Figure 11. Inlet airflow velocity profile

\subsection{Theoretical Equations and Parameters}

For comparison with previous study, the theoretical equations and parameters were used in the same manner. 
The Reynolds number was applied as a relevant number and it takes a form as

$$
\mathrm{Re}=\frac{c L}{v}
$$

where $\mathrm{c}[\mathrm{m} / \mathrm{s}]$ is the airflow velocity, $\mathrm{L}[\mathrm{m}]$ is characteristic length and $v\left[\mathrm{~m}^{2} / \mathrm{s}\right]$ is a kinematic viscosity of the surrounding air. The characteristic length is defined as equivalent hydraulic diameter

$$
L=\frac{4 A}{O}
$$

where $A\left[\mathrm{~m}^{2}\right]$ is a tunnel cross section and $\mathrm{O}[\mathrm{m}]$ is circumference of the tunnel cross-section. To compare results with other studies every physical quantity was normalized. For temperature evaluations, the normalization to ambient temperature was used. The normalized temperature is defined as

$$
T_{N}=\frac{T}{T^{*}}
$$

where $T$ is measured temperature and $T^{*}$ is ambient temperature during the measurement. Assuming, that the lab space was large enough, the heat power could not influence the internal temperature in the surroundings. It means that this ambient temperature corresponded to the temperature of the surroundings. The heat transferred throughout the sheets can be expressed as follows

$$
Q_{S}=Q_{C}-Q_{Z}
$$

where $Q_{C}$ is the overall heat generated by the heating wire and $Q_{Z}$ is the heat loss through heating box walls. The heat loss is determined by heating box materials. The transferred heat from the sheet surfaces to the surrounding airflow is defined as

$$
Q_{S}=\alpha\left(t-t_{s}\right) S
$$

where $\alpha$ is the convective heat transfer coefficient, $t$ is average sheet temperature, $t_{s}$ is ambient temperature and $S$ is the sheet area. In case of convection, the heat transfer intensity is commonly express by the Nusselt number, which takes a form as

$$
N u=\frac{\alpha l}{\lambda}
$$

Where 1 is the sheet length and $\lambda$ is the thermal conductivity of the surrounding air.

\section{RESULTS}

Dependence of the sample's surface average temperature to airflow velocity is shown in Figure 12. and in Figure 13. for surface maximal temperature. The results are combined with results of steel sample from previous study. Results shown that composite sample achieved lower both average and maximum temperatures than steel sample. For lower power inputs the temperature dependence on Reynolds number was similar to steel sample. For higher power inputs the dependence on composite sample was deformed in comparison with steel.

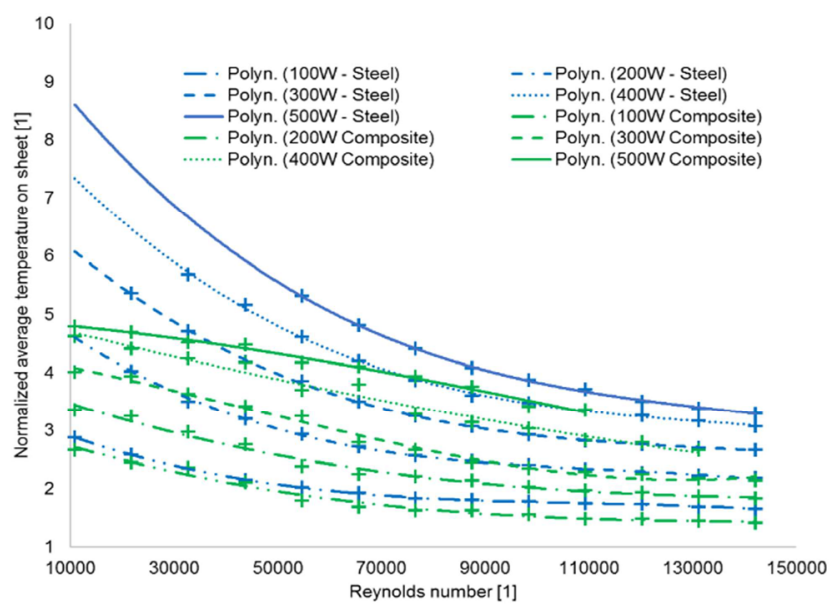

Figure 12. Comparison of average temperatures on sheets surfaces

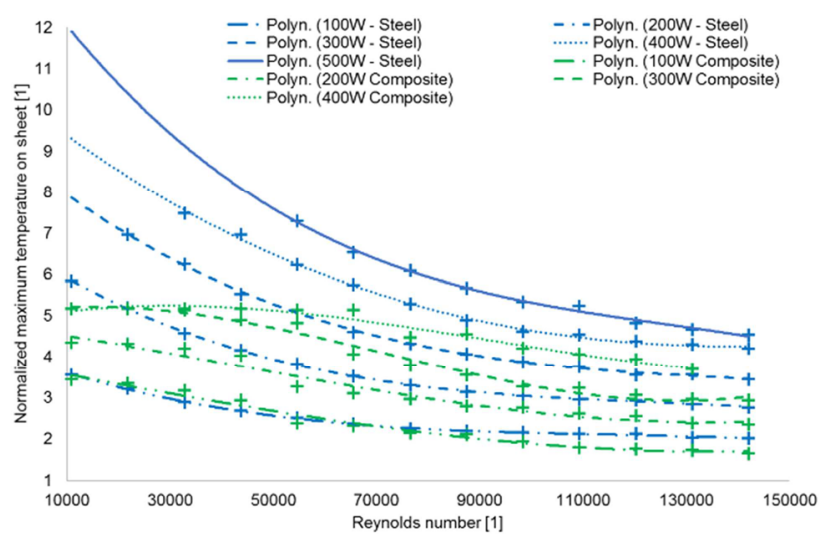

Figure 13. Comparison of maximum temperatures on sheets surfaces

Infrared image samples comparing the temperature distribution of composite and steel sample are shown in Figure 14. and Figure 15. Temperature distribution over composite sample is more homogenous. Distribution field is similar to previous results from numerical simulation described in [3]. For comparison, the image is shown in Figure 16. Unfortunately, the numerical simulation was realized for higher Reynolds number so it is not possible to compare in terms of temperature values, but only in terms of temperature distribution. 


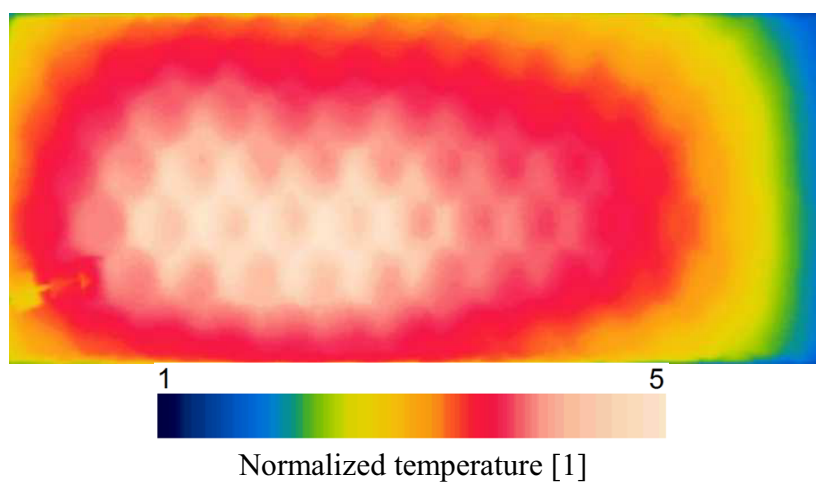

Figure 14 : Temperature distribution on steel sample $300 \mathrm{~W}, 5 \mathrm{~m} / \mathrm{s}$

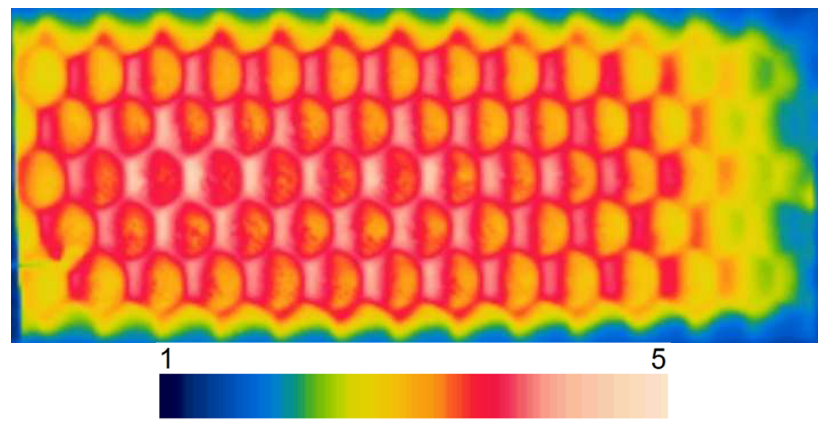

Normalized temperature [1]

Figure 15 : Temperature distribution on composite sample $300 \mathrm{~W}, 5 \mathrm{~m} / \mathrm{s}$

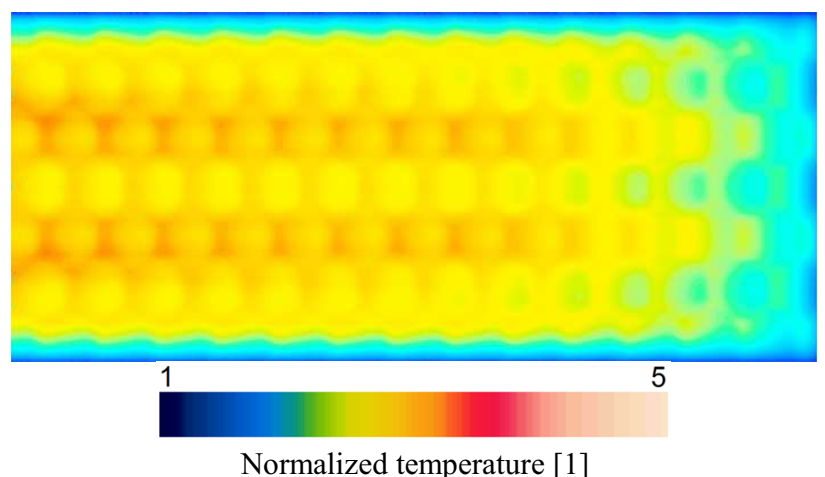

Figure 16 : Numerical simulation of temperature distribution on steel sample $300 \mathrm{~W}, 13 \mathrm{~m} / \mathrm{s}$

\section{Conlusion}

Based on the structural metal sheets, several samples from carbon and glass fabrics were created. To obtain an accurate $3 \mathrm{D}$ CAD models of this newly created parts, using of reverse engineering methods, namely the 3D scanning it was necessary. One of possible way, how to get the real geometry based on manual repairing of the scanned data by the vector blends definition is described. In respect to future numerical modeling the dependence of the geometry parameters to created mesh were compared. That could be even better and easier to use coarse geometry instead of highly optimized and smoothed one has been found.

Results of composite sample shown that temperatures on the sample was lower than in case of steel sample. This leads to conclusion that composite sample reached the better heat transfer than steel sample. This trend was not expected because with same surface structure the heat transfer of composite material should be lower than heat transfer of steel. It is possible that composite sample caused different airflow behaviour near the surface because the surface was not glossy as the steel sample, but it was covered by small roughness from the production. This could lead to higher turbulence intensity and higher heat transfer. For confirmation, it is planned to repeat the measurement. The fact that composite sample shown better homogeneity of temperature distribution than steel one will be studied in future research. Study also shown, that in conditions of higher ambient temperature the plastic made aerodynamics tunnel is not solid to stand under pressure in higher Reynolds number. This finding will be considered for future development of the experimental device. Also, numerical modeling of materials reinforced with fibers and dependency on the plies angles, numbers and alignment would be carried.

This publication was written at the Technical University of Liberec as part of the project "Innovation of technical systems structures with the use of composite materials" with the support of the Specific University Research Grant, as provided by the Ministry of Education, Youth and Sports of the Czech Republic in the year 2016 .

\section{References}

1. V. Malikov, Umformung von strukturieiten Blechen. Winter-Industries, 105 (2013)

2. Simon S., Werkstoffgerechtes Konstruieren BTU Cottbus, 208 (2005)

3. J. Egert, K. Frana, S. Simon, S. Wichmann, Heat Transfer Studies on Structured Metal Sheets. KMUTNB: IJAST, 9, (2016)

4. Sterzing A., Bewertung von Leichtbaupotenzial Zwickau: Drecht aus dem IWU, (2005)

5. Thill C., Etches J.A., Bond I.P., Trapezoidal corrugated laminates, Appl. Science, 41, (2010)

6. K. Frana, S. Simon, Air Flows along Perforated Metal Plates, Journal of Mech, 9, (2015)

7. R. L. Webb, N. Kim, Principles of enhanced heat transfer, Taylor \& Francis, (2005)

8. P. Kulhavy, J. Petrik, P. Srb, P. Lepsík: Vibration response of composite structures, EAN 2016, Uni. of West bohemia, (2016)

9. W. T. Wang, T. Y. Kam, Material characterization of laminated composite plates, Composite Structure, 50 (2000)

10. D. Briassoulis, Equivalent orthotropic properties of corrugated sheets, Comp. \& Structures 23, (1986)

11. ANSYS, Inc., Mechanical Structural Nonlinearities Meshing in mechanical, (2010)

12. P. Kulhavy, P. Srb, P. Lepsík: Digitization of structured composite, EVM, Ústí n. L., 4, (2016) 